\title{
frazzled Encodes a Drosophila Member of the DCC Immunoglobulin Subfamily and Is Required for CNS and Motor Axon Guidance
}

\author{
Peter A. Kolodziej, ${ }^{\star}+$ Leslie C. Timpe, ${ }^{\star}$ \\ Kevin J. Mitchell,‡ Sharon R. Fried,* \\ Corey S. Goodman, $¥$ Lily Yeh Jan, ${ }^{*}$ and Yuh Nung Jan* \\ ${ }^{*}$ Howard Hughes Medical Institute \\ Department of Physiology \\ Department of Biochemistry \\ Third and Parnassus Avenues \\ University of California, San Francisco \\ San Francisco, California 94143-0724 \\ †Howard Hughes Medical Institute \\ Department of Cell Biology \\ Vanderbilt University Medical Center \\ Nashville, Tennesee 37232-0295 \\ ¥Howard Hughes Medical Institute \\ Division of Neurobiology \\ Department of Molecular and Cell Biology \\ University of California, Berkeley \\ Berkeley, California 94720
}

\section{Summary}

We have identified a Drosophila member of the deleted in colorectal cancer (DCC) gene family. The frazzled gene encodes transmembrane proteins that contain four immunoglobulin C2 type domains, six fibronectin type III repeats, and a cytoplasmic domain of $\mathbf{2 7 8}$ amino acids. Like vertebrate members of the DCC family, Frazzled is expressed on axons in the embryonic central nervous system and on motor axons in the periphery. Frazzled is also expressed on epidermis and gut epithelium. Null mutants in frazzled are defective in axon guidance in the central nervous system and in motor axon guidance and targeting in the periphery. The phenotypes strongly resemble those of a deletion of the two Drosophila Netrin genes. We have rescued the frazzled CNS and motor axon defects by expressing Frazzled specifically in neurons; expression in target tissues does not rescue the phenotype. These data, together with vertebrate studies showing binding of DCC to netrin, suggest that Frazzled may function in vivo as a receptor or component of a receptor mediating Netrin-dependent axon guidance.

Introduction

Secreted and membrane-bound guidance cues can function as either attractants or repellents to influence axon pathfinding (reviewed by Tessier-Lavigne, 1994; Goodman, 1996), and these molecules appear to be highly conserved among invertebrates and vertebrates. For example, netrins, secreted proteins related to laminin, are expressed at the midline in a variety of organisms, where they function via as yet unidentified receptors as both chemoattractants and chemorepellents (Hedgecock et al., 1990; Ishii et al., 1992; Kennedy et

§ Present address: Cardiology Research 111C5, Veterans Affairs Center, 4150 Clement Street, San Francisco, California 94121. al., 1994; Serafini et al., 1994; Colamarino and TessierLavigne, 1995; Harris et al., 1996; Mitchell et al., 1996; Wadsworth et al., 1996). Growth cone responses to these and other candidate guidance molecules vary with neuronal cell type, suggesting that the distribution and diversity of both the guidance ligands and their receptors play a key role in determining the patterns of neuronal connections.

The Deleted in Colorectal Cancer (DCC) protein and the related neogenin protein are immunoglobulin (Ig) superfamily members that are expressed on developing axons. These two molecules share $\sim 52 \%$ amino acid identity and belong to a distinct Ig subfamily. Their extracellular domains contain four immunoglobulin C2 repeats and six fibronectin III repeats (Hedrick et al., 1994; Vielmetter et al., 1994) and their $\sim 300$ amino acid cytoplasmic domains show no significant homology to proteins outside the subfamily (Hedrick et al., 1994; Vielmetter et al., 1994).

The deleted in colorectal cancer gene was originally isolated as a possible tumor suppressor (Fearon et al., 1990 ), but the role of $D C C$ in tumor progression and normal development is unclear. Both DCC and neogenin are also expressed in most epithelial tissues during development, particularly in the gut. The structure of DCClike molecules and the timing of their expression during development of the nervous system and gut has led to proposals that they function as negative regulators of cell division or as receptors for morphogenetic information (Chuong et al., 1994; Hedrick et al., 1994; Pierceall et al., 1994; Vielmetter et al., 1994). Either role could explain why the loss of $D C C$ appears to promote tumor progression, since tumors are abnormal in both cell growth and morphology.

We have identified a Drosophila gene, frazzled (fra), that encodes a DCC-related protein. Frazzled is also expressed on axons in the developing nervous system, as well as on a variety of epithelia. Null mutations in fra disrupt axon guidance in the central nervous system and motor axon pathfinding and target recognition in the periphery. The phenotypes strongly resemble those observed in embryos lacking the two Drosophila Netrin genes (Harris et al., 1996; Mitchell et al., 1996). We also provide evidence that fra functions cell autonomously, raising the possibility that it encodes a receptor or part of a receptor mediating Netrin-dependent guidance. Together with the observation that DCC and neogenin are vertebrate netrin receptors (Keino-Masu et al., 1996 [this issue of $\mathrm{Ce} /[$ ) and studies on a DCC-related protein, UNC-40, in C. elegans (Chan et al., 1996 [this issue of Cel/]), these data suggest that mechanisms of netrindependent axon guidance are evolutionarily conserved.

\section{Results}

The frazzled Gene Encodes DCC-Related Proteins We identified a $P$-lac $Z$ insertion at $49 B$ in an enhancer trap screen for mutations that affect nervous system development and function (Bier et al., 1989). The locus 
A.

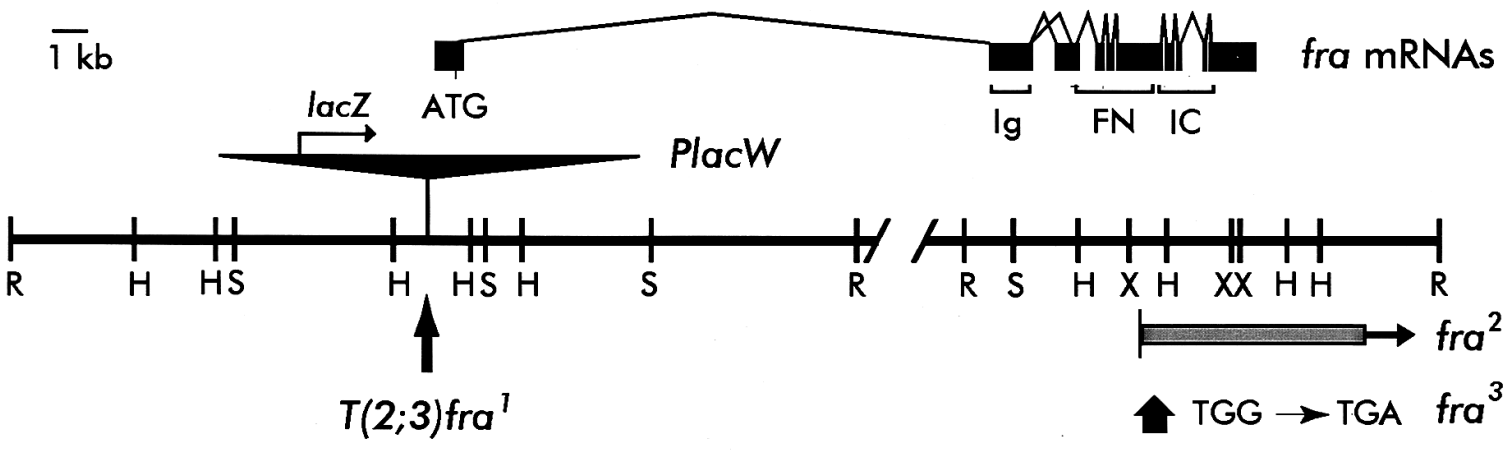

B.

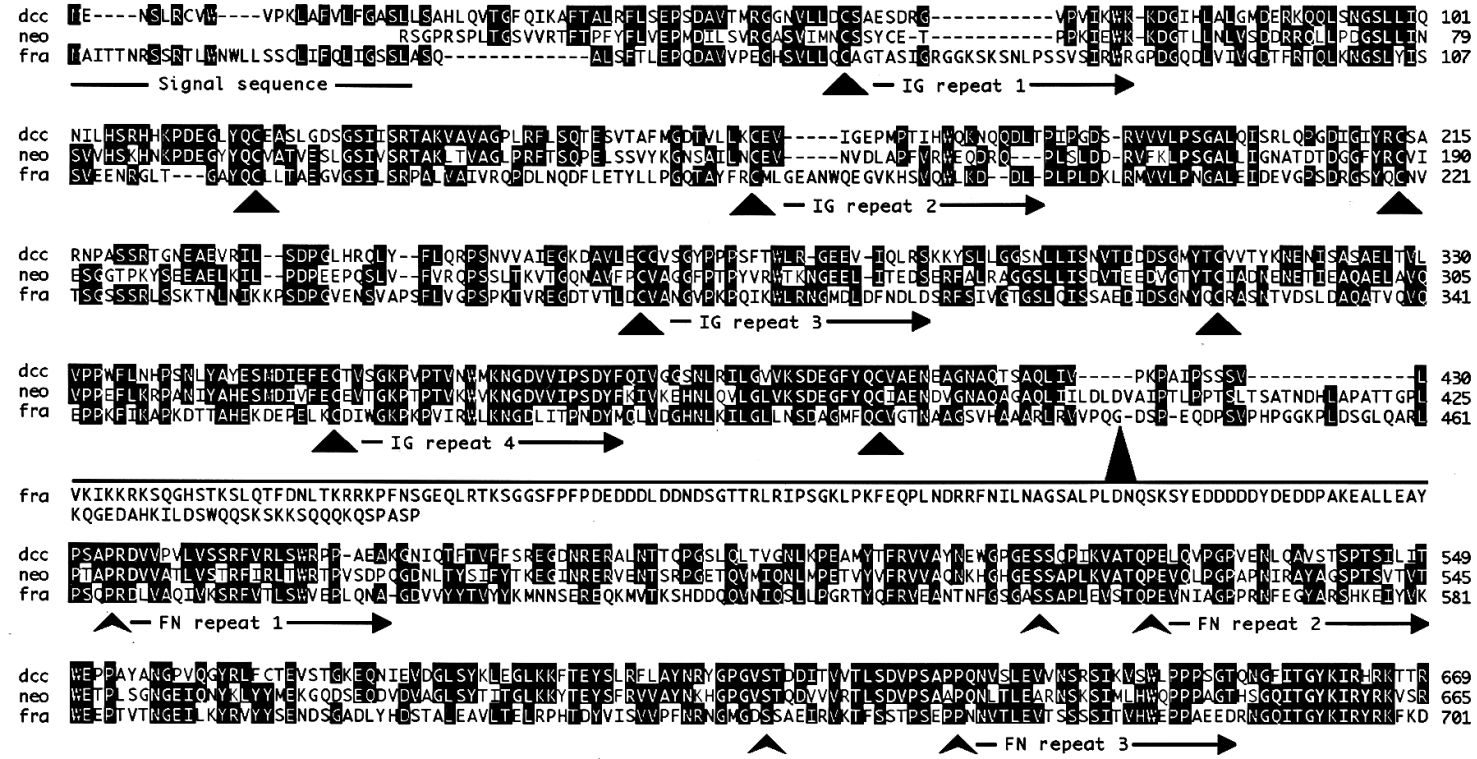

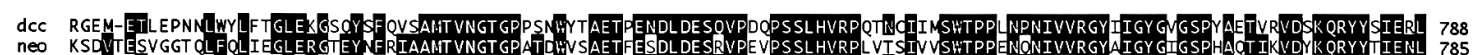

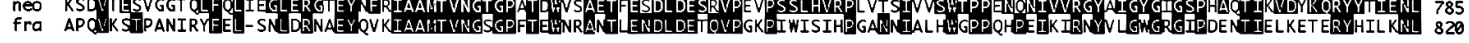
A $\quad$ - FN repeat $4 \longrightarrow$

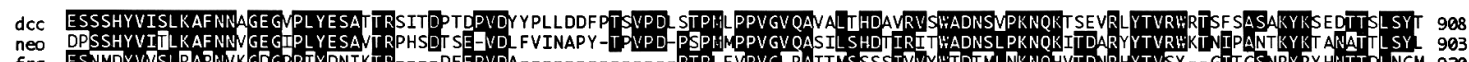

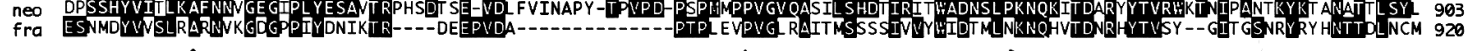

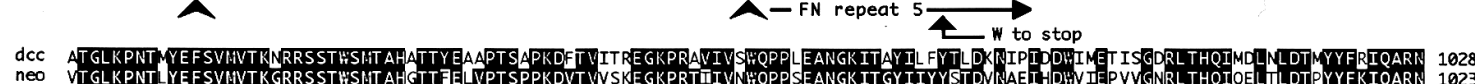

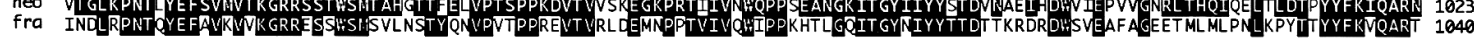
A 1 FN repeat $6 \longrightarrow$

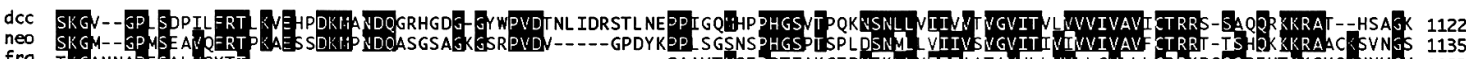

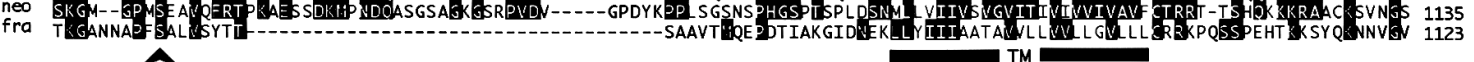

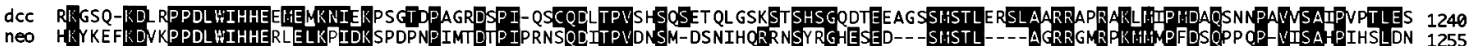

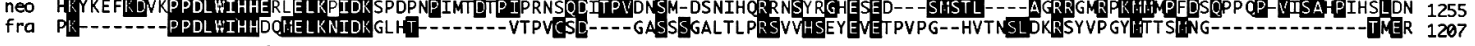

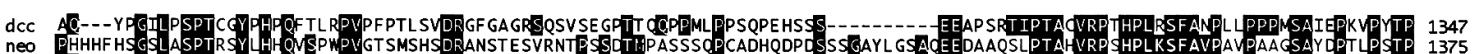

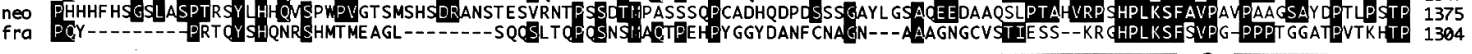

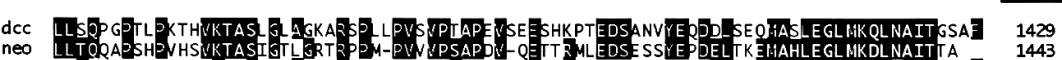

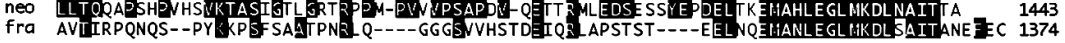

Figure 1. Genomic Organization of the frazzled (fra) Locus and Sequence Identities among DCC Family Members (A) The bottom line represents a partial restriction map of the genomic region immediately flanking the P-element and encoding the fra transcripts. The probes used to seek transcripts in the vicinity of the insertion site were derived by labeling restriction fragments that span the region. Northern analysis and in situ hybridization with the $2.3 \mathrm{~kb}$ HindllI DNA fragment containing the P-element insertion site detected 
was named frazzled (fra) because adult flies homozygous for the P-element shake upon revival from etherinduced anesthesia. Precise excision of the $P$ element reverted the phenotype, thus establishing that the shaking defect is due to the insertion. Other excision lines were homozygous embryonic lethals that failed to complement each other and the existing deficiency for 49B, $D f(2 R) v g 135$. Since embryos bearing the 49B enhancer trap expressed lacZ in the CNS (data not shown), we investigated whether a nearby gene might play an essential role in nervous system development or function.

In situ hybridization experiments on embryos with probes covering $\sim 10 \mathrm{~kb}$ of DNA flanking the $P$-lacZ insertion identified a single transcription unit in the region. frazzled cDNA clones encode two isoforms that exhibit $\sim 43 \%$ overall sequence identity to $D C C$ and neogenin (Figure 1) and the expression pattern of frazzled transcripts matches that of the enhancer trap (data not shown).

Frazzled, DCC, and neogenin belong to the same Ig subfamily. The extracellular domains of the two predicted Frazzled isoforms contain four immunoglobulin (Ig) C2 type repeats followed by six fibronectin repeats, as do those of DCC and neogenin (Hedrick et al., 1994; Vielmetter et al., 1994). The two isoforms differ by an insertion of 151 amino acids between the fourth immunoglobulin repeat and the first fibronectin repeat (Figure 1). They share a membrane-spanning domain and a cytoplasmic domain that is $\mathbf{2 7 8}$ amino acids in length. Within the cytoplasmic domain, there are several regions of amino acid identity with the vertebrate proteins (Figure 1); no other close matches were found in public sequence databases.

\section{Frazzled Is Expressed on Extending Axons}

during Development

Antisera that specifically recognize the Frazzled C-terminal domain reveal that, like DCC and neogenin, Frazzled is expressed on developing axons and epithelia in the embryo. Frazzled is expressed at high levels on commissural and longitudinal axons in the developing CNS (Figure 2) and is detected at stage 13 on the earliest commissural axons (data not shown). Frazzled is present at lower levels on peripheral motor axons that extend outward in the intersegmental and segmental nerves (Figure 2 and data not shown) and also on the surfaces of midgut epithelial cells beginning at stage 12 and on epidermis (data not shown). Frazzled does not appear to be expressed on tissues that are thought to express ligands required for motor and CNS axon pathfinding
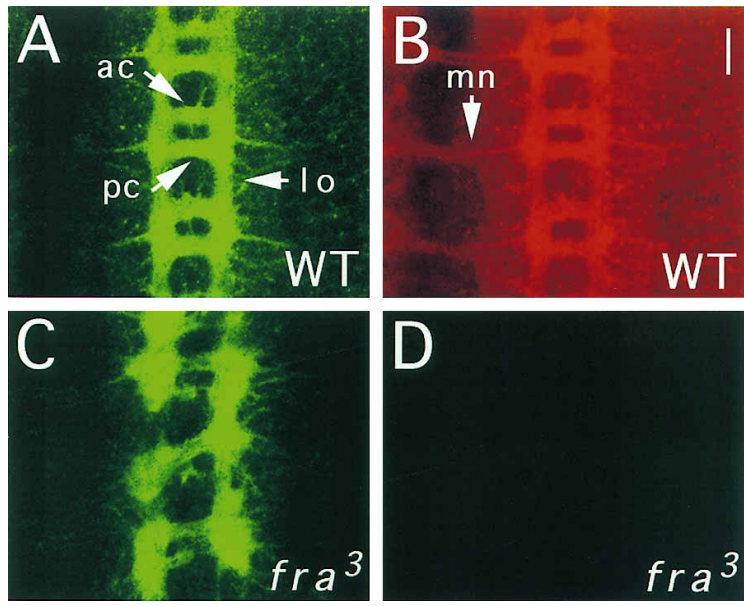

Figure 2. Frazzled Is Expressed on CNS Axons

(A) Commissural and longitudinal axons in the CNS of a stage 15 wild-type embryo visualized with MAb BP102. The axon pathways form a regular ladder-like pattern, with commissural axons forming the rungs, and longitudinal axons forming the sides. ac, anterior commissure; pc, posterior commissure; and lo, longitudinal axons. (B) The same embryo as in A), but stained with anti-Frazzled C-terminal domain. Frazzled is detected on commissural and longitudinal axons and on motor axons $(\mathrm{mn})$ projecting from the CNS. (C) Commissural and longitudinal axons in the CNS of a stage 15 fra $^{3}$ embryo visualized with MAb BP102. The commissural axons are absent or reduced in number, and gaps appear in the longitudinal axon tracts.

(D) The same embryo as in (C), but stained with anti-Frazzled. Frazzled is not detected in $\mathrm{fra}^{3}$ embryos, or in $\mathrm{fra}^{1}, \mathrm{fra}^{2}$, or $\mathrm{fra}^{4}$ mutant embryos (data not shown).

Confocal images are from a $1 \mu \mathrm{m}$ thick optical section. Anterior is at the top here and in Figure 3.

such as muscle, glia, or midline cells (Figure 2 and data not shown). Frazzled immunoreactivity is absent in homozygous $\mathrm{fra}^{1}, \mathrm{fra}^{2}, \mathrm{fra}^{3}, \mathrm{fra}^{4}$ mutant embryos (Figure 2 and data not shown).

\section{Null Mutations in fra and NetrinA/B Similarly Affect CNS Axon Development and Motor Axon Pathfinding}

To investigate the role of DCC-like proteins in development, we identified null mutants in fra among embryonic lethal lines generated either by imprecise excision of the P-element inserted adjacent to the fra transcription

an $\sim 6 \mathrm{~kb}$ message that was abundantly expressed in the CNS and gut epithelium. Other probes did not identify additional transcripts. The positions of the $\mathrm{fra}^{1}$ translocation, the $\mathrm{fra}^{2}$ deletion breakpoints and the fra ${ }^{3}$ point mutation are indicated. The fra ${ }^{2}$ deletion extends $3^{\prime}$ beyond the fra gene. The thick blocks in the top line indicate the relative size and positions of the ten exons in the identified fra mRNAs; the gaps indicate the relative sizes of the nine introns. The actual size of the first intron is unknown. The exons corresponding to the four immunoglobulinlike (Ig) repeats, the six fibronectin type III (FN) repeats, and the intracellular (IC) domain are indicated by brackets.

(B) fra encodes two DCC-related proteins 1355 and 1506 amino acids in length. BLAST (Altschul et al., 1990) was used to identify DCC, neogenin, and Frazzled as related, and the sequences were aligned (Higgins, 1994). Identical residues are shaded. The four Ig domains are shown and triangles indicate the position of the two conserved cysteines present in each domain (Williams and Barclay, 1988). Arrowheads indicate the amino acids at the ends of each fibronectin type III repeat (Patthy, 1990). The longer fra cDNA encodes a 151 amino acid sequence not found in other DCC-like proteins. The putative signal sequence, transmembrane region (TM), and three areas of particularly strong identity among the predicted intracellular domains of human DCC, chick neogenin, and Frazzled are underlined. The sequence alteration in the fra ${ }^{3}$ allele changes the TGG encoding W 1028 to TGA, a stop codon. 
start site or by ethyl methanesulfonate (EMS) mutagenesis (Experimental Procedures and Figure 1). Since the $\mathrm{fra}^{3}$ and $\mathrm{fra}^{4} \mathrm{EMS}$ alleles were obtained in two different genetic backgrounds, we focused our investigations on the development of Frazzled expressing CNS and motor axons in transheterozygous $\mathrm{fra}^{3} / \mathrm{fra}^{4}$ mutant embryos.

The CNS in each segment of wild-type embryos stained with MAb BP102 (Seeger et al., 1993) contains bilaterally symmetric longitudinal tracts and two commissural tracts (anterior and posterior), all of which express Frazzled. In fra mutant embryos, partially penetrant defects are observed in the earliest stages of the development of the commissures. In $\mathrm{fra}^{3} / \mathrm{fra}^{4}$ mutant embryos, $12 \%$ of anterior commissures and $43 \%$ of posterior commissures $(n=182)$ in abdominal segments A1-A7 are thin or absent. Commissures that appear to be relatively normal in thickness are often less wellorganized than normal (Figures 2 and 3). Comparable results are observed in other fra alleles (data not shown). Occasional breaks are also observed in the longitudinal tracts (Figures 2 and 3).

Approximately 40 motor axons in each abdominal hemisegment of the Drosophila embryo extend into the periphery where they innervate 30 body wall muscles in a highly stereotyped pattern. A subset of motor axons exit the ventral CNS in the intersegmental nerve (ISN) and extend dorsally to innervate the NetrinA/B expressing dorsal muscles 1 and 2 (Mitchell et al., 1996) (Figure 4). In fra mutant embryos, these ISN axons, which normally express Frazzled (Figure 2 and data not shown), extend dorsally, but often branch or extend inappropriately when they reach the dorsal muscle region. In $20 \%$ of hemisegments (143 scored), these ISN axons wander into adjacent segments or toward the dorsal midline, appear to make contacts with inappropriate muscles, or branch more extensively over their normal muscle targets (Figure 4). The projections of motor axons in the segmental nerve b (SNb) to ventral muscles 6 and 7 , which express $N e t B$, are also occasionally aberrant in fra embryos (Figure 4). It is possible, however, that the $\mathrm{SNb}$ innervation defects, unlike the ISN defects, are secondary to previous routing errors made by these axons in the CNS. ISN axons exit the CNS normally in fra embryos, but $\mathrm{SNb}$ axons must first cross the midline.

The CNS defects and ISN motor axon defects in fra null mutant embryos strongly resemble those observed in embryos homozygous for a deletion on the $X$ chromosome that removes the two tandem Drosophila Netrin genes (Harris et al., 1996; Mitchell et al., 1996). In Netrin $A / B$ mutant embryos, the posterior commissure is also more severely affected than the anterior commissure (Figure 3). Occasional breaks in the longitudinal tracts are also observed in the Netrin double mutant. Moreover, in Netrin mutant embryos, the ISN axons display a similar frequency of dorsal muscle targeting errors and innervation of muscles 6 and 7 by the SNb motor axons is similarly affected (Mitchell et al., 1996). In fra and Netrin mutants the $\mathrm{SNa}$ axons project normally to their lateral muscle targets, which do not normally express Netrin (Figure 4). However, these axons do express Frazzled and their trajectory can be altered by ectopic Netrin expression on all muscles (Mitchell et al., 1996).

The fra mutant phenotypes do not appear to be due
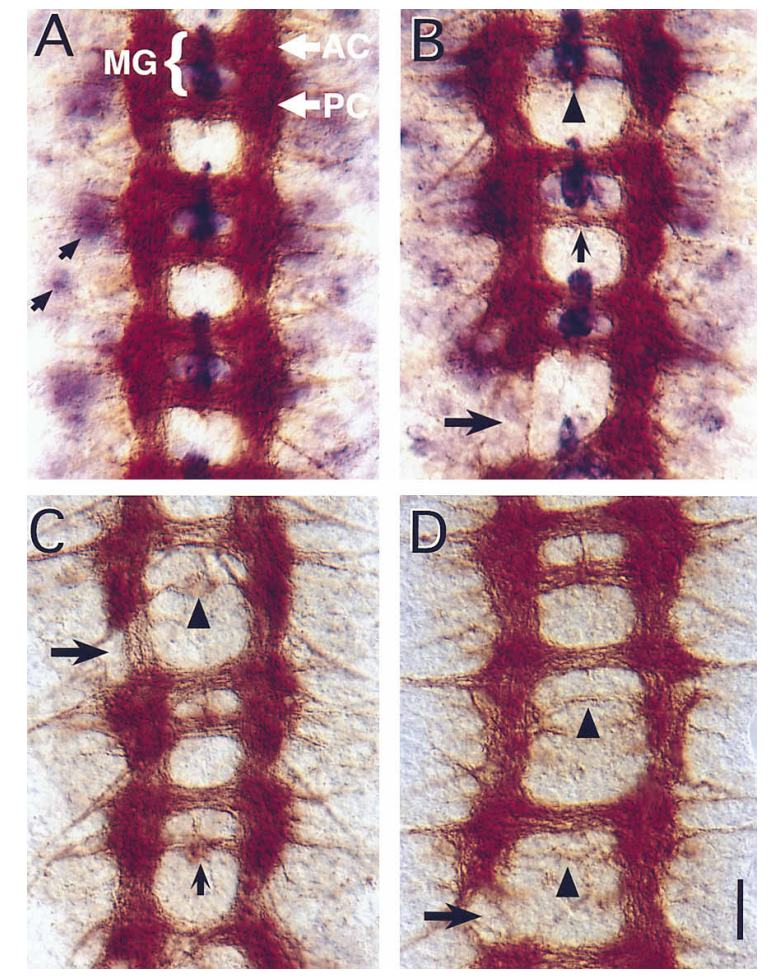

Figure 3. frazzled Is Required for Commissural Axons to Project toward Netrin-Expressing Midline Cells

(A) Expression of Netrin mRNAs (purple) in the CNS of a wild-type embryo. Netrins $A$ and $B$ are expressed at high levels by the midline glia (MG) and at lower levels by lateral clusters of CNS neurons (black arrows). AC, anterior commissure; PC, posterior commissure. (B) Expression of Netrins is normal in a fra $^{3} /$ fra $^{4}$ mutant embryo, but the posterior commissures are thinner (small arrow) or absent (arrowhead) (except for the median fascicle projecting out from the midline neurons). A break has occurred in the longitudinal tracts in one segment (large arrow). The anterior commissures also appear thinner than normal.

(C) Another fra ${ }^{3} / f r a^{4}$ embryo stained with MAb BP102. Some posterior commissures are considerably thinner (small arrow) or absent (arrowhead), the anterior commissures are slightly thinner than normal, and the longitudinal tracts are interrupted (large arrow).

(D) Similar commissural and longitudinal axon tract defects are observed in embryos hemizygous for the deficiency (Df(1)NP5) that removes both Netrin genes (Mitchell et al., 1996). Arrowheads, missing posterior commissures; arrow, longitudinal break.

Bar, $10 \mu \mathrm{m}$.

to alterations in the survival, fate, or patterning of midline cells, CNS neurons, muscle, or motor axons. In addition to BP102, numerous markers for CNS development (Engrailed, Eve, Ftz, Connectin, 22C10, Fasciclin II) are expressed normally in frazzled mutants (data not shown) and midline cells appear to express normal levels of mRNA of NetrinA and NetrinB (Figure 3).

\section{Expression of Frazzled in Neurons, but Not in Target Tissues, Rescues the fra CNS and Motor Axon Guidance Phenotypes}

We investigated whether expression of a fra cDNA in all neurons in fra mutant embryos could restore the abilities of commissural axons to cross the midline and ISN motor axons to find their dorsal muscle targets. As a control, we first tested whether overexpression of the shorter 

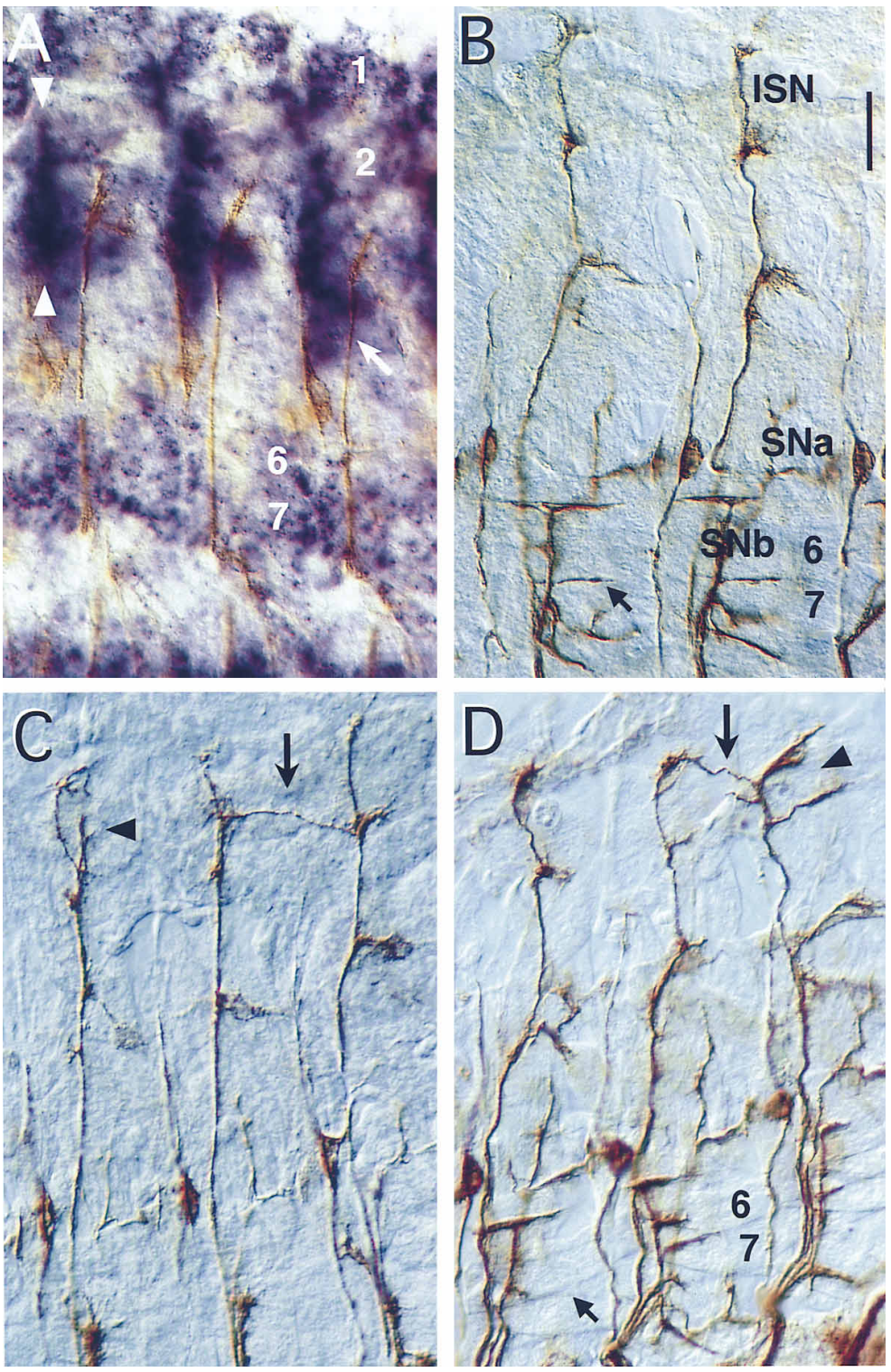

Figure 4. frazzled Is Required for Motor Axon Pathfinding to Netrin-Expressing Target Muscles

(A) A stage 15 embryo double-stained with MAb 1D4 (brown) (Van Vactor et al., 1993) and antisense probes to Netrins $A$ and $B$ (purple). The ISN (white arrow) is growing out dorsally, just posterior and internal to the epidermal stripe of NetrinA expression (white arrowheads) toward the dorsal muscle group. Dorsal muscles 1 and 2 express Netrins just prior to their innervation by ISN motor axons, and ventral muscles 6 and 7 express NetrinB (Mitchell et al., 1996).

(B) The pattern of muscle innervation in a wild-type embryo (late stage 16). The ISN has contacted the dorsal muscles, the $\mathrm{SNa}$ (forked structure, out of focus) projects to the lateral muscles, and the SNb innervates the ventral muscles. Innervation in the synaptic cleft between muscles 6 and 7 is indicated by an arrow.

(C) The pattern of muscle innervation in a fra $^{3 /}$ fra $^{4}$ mutant embryo (early stage 16). ISN motor axons make inappropriate contacts with dorsal muscles (arrowhead) or extend a collateral branch into an adjacent segment (concave arrow).

(D) The pattern of muscle innervation in a $\mathrm{fra}^{3}$ / fra $^{4}$ mutant embryo (late stage 16). ISN motor axons branch excessively and make inappropriate contacts with dorsal muscles (arrowhead) or project into adjacent segments (concave arrow). Ventral muscles 6 and 7 that lack $\mathrm{SNb}$ innervation in one segment are shown (small arrow).

Bar, $15 \mu \mathrm{m}$.
Frazzled isoform in all neurons could perturb axon development in otherwise wild-type embryos. We crossed flies in which the shorter cDNA was driven by the GAL4 UAS to the 1407 GAL4 line in which all neurons express the GAL4 transcription factor (Brand and Perrimon, 1993; Luo et al., 1994). 1407-GAL4/+; UAS-fra/+ embryos express Frazzled on all axons; however, no defects in CNS or PNS projections are observed with MAbs BP102, 22C10, or 1D4, and viable adult flies are recovered (Figure 5 and data not shown).

We created flies containing the UAS-fra transgene and a second chromosome bearing the 1407 GAL4 enhancer trap and $f^{3}{ }^{3}$ and crossed them to fra $^{4}$ flies. We identified transheterozygous fra mutant embryos as those lacking marked balancers; half of these embryos expressed Frazzled strongly in all neurons, but not in epithelia or gut. Commissures appear to form normally in these rescued fra embryos $(n=70$ anterior and 70 posterior commissures in abdominal segments A1-A7), but not in mutant embryos lacking the UAS-fra transgene (Figure
5). In rescued fra embryos, the ISN motor axons also innervated their targets at near wild-type levels (95/98 hemisegments scored) (Figure 5). Thus, this Frazzled isoform can function in neurons to promote proper axon extension across the midline and motor axon targeting to netrin expressing muscles.

We then used the pan-muscle GAL4 line 24B (Luo et al., 1994) to test whether Frazzled was acting cell autonomously in motor axon targeting. We found that expression of Frazzled in all muscles in fra mutant embryos neither rescues nor enhances fra motor axon defects (Figure 5), and does not affect axon targeting in wild-type embryos (data not shown). From these experiments and the observed normal expression of Frazzled in CNS and motor axons, but not in nonneuronal tissues that affect pathfinding (midline cells and glia), we believe that Frazzled is likely to act as a receptor or component of a guidance receptor.

Not all fra CNS defects were rescued. Rescued embryos exhibited 1-3 gaps per embryo in the longitudinal 

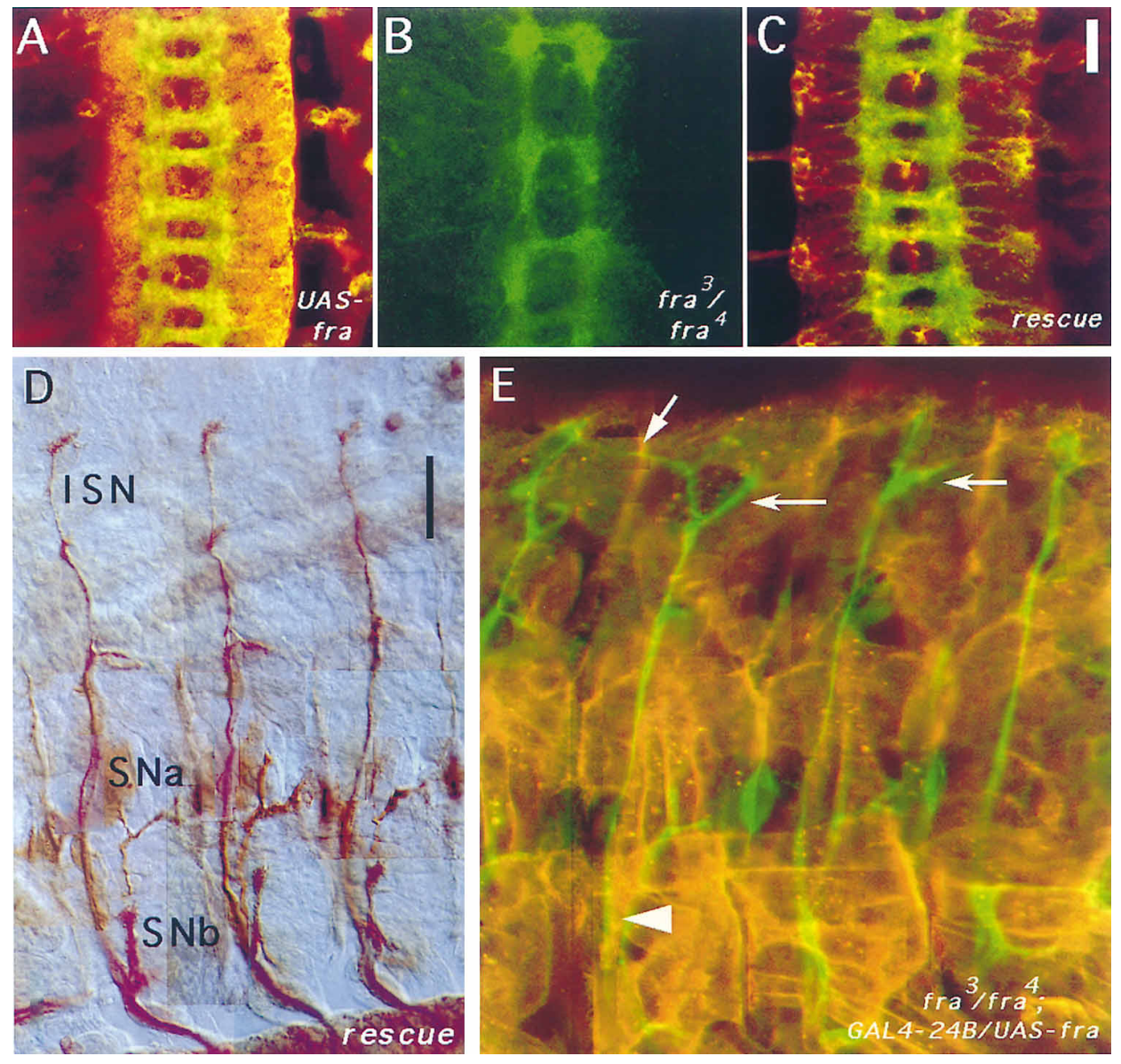

Figure 5. Expression of the Shorter Frazzled Isoform in All Neurons, but Not in Target Tissues, Rescues Commissure and Motor Axon Guidance Defects

All embryos were stained with rabbit anti- $\beta$-galactosidase (1:1000), rabbit anti-Frazzled C-terminal domain (1:1500), and either mouse MAb BP102 (1:20) or 1D4 (1:5).

(A) Commissures (green, MAb BP102) form normally in a GAL4-1407 fra ${ }^{3} / C y O-e l a v-l a c Z$ (wild-type) embryo. The shorter Frazzled isoform and $\beta$-galactosidase (both in red) are expressed in all neurons.

(B) Commissures are absent or thinned in GAL4-1407 $\mathrm{fra}^{3} /+\mathrm{fra}^{4}$ embryos. Frazzled and $\beta$-galactosidase staining are absent.

(C) Commissure formation is restored in GAL4-1407 $\mathrm{fra}^{3} /+\mathrm{fra}^{4}$; UAS-fra/+ embryos. The shorter Frazzled isoform (red) is expressed in all neurons; $\beta$-galactosidase staining is absent. Bar for $(A)-(C), 10 \mu \mathrm{m}$.

(D) Restored dorsal muscle innervation in a GAL4-1407 fra $3 /+$ fra $^{4}$; UAS-fra/+ embryo (early stage 16; SNb has not fully developed). The CNS is dark due to the expression of Fra in all neurons. Bar for (D) and (E), $15 \mu \mathrm{m}$.

(E) Motor axons (green) branch excessively (concave arrows) or cross segments (arrow) in a fra 3 fra $^{4}$; GAL4-24B/UAS-fra embryo that expresses Frazzled (red) in all muscles. SNb axons are absent in one hemisegment (arrowhead).

axon tracts. These remaining defects may reflect a requirement for other Frazzled isoforms in the formation of these tracts or other differences between transgene and wild-type frazzled gene expression.

\section{Discussion}

We have identified a Drosophila DCC-related gene called frazzled, and demonstrated that this Ig superfamily member is expressed on and required for the pathfinding of Netrin-responsive axons. We have also provided evidence that it mediates guidance cell autonomously by rescuing the defects in motor axon targeting by expression in neurons, but not by expression in muscle. Although we have not shown directly that Frazzled binds Netrin proteins, these data and Frazzled similarity to DCC, a vertebrate netrin receptor (Keino-Masu et al., 1996), strongly suggest that Frazzled is a receptor or a ligand-binding component of a Drosophila Netrin receptor. A parallel study in C. elegans (Chan et al., 1996) shows that UNC-40 encodes a DCC-related protein and has a mutant phenotype that overlaps with the netrin unc-6 phenotype (Hedgecock et al., 1990). These results indicate that the mechanisms underlying netrin-dependent axon guidance are likely to be conserved among vertebrates, flies, and nematodes.

\section{frazzled and Other DCC Family Members May Encode Netrin Receptors} Netrins are secreted, laminin-related proteins that are expressed by midline cells and other tissues in worms, fruit flies, and vertebrates. Netrins are required in vivo 
for commissural axon guidance (Hedgecock et al., 1990; Harris et al., 1996; Mitchell et al., 1996) and are chemoattractants for commissural axons in vitro (Kennedy et al., 1994; Serafini et al., 1994). Netrins can also repel certain axons in vitro (Colamarino and Tessier-Lavigne, 1995) and most likely in vivo as well (Hamelin et al., 1993; Wadsworth et al., 1996).

Analysis of mutations removing the two Drosophila Netrin genes (NetrinA and NetrinB) identifies commissure formation and ISN dorsal muscle targeting as Netrin-dependent guidance processes (Harris et al., 1996; Mitchell et al., 1996). Midline and muscle expression of $N$ etrinA/B is appropriately placed and timed and is required to steer approaching Frazzled-expressing axons.

frazzled null mutants disrupt guidance events that also depend on NetrinA/B, but do not have other guidance phenotypes seen in other CNS or motor axon mutants (Seeger et al., 1993; Van Vactor et al., 1993; Kolodziej et al., 1995). Among the extensive collections of mutants affecting guidance in the CNS (Seeger et al., 1993; Kolodziej et al., 1995), the fra phenotypes most closely resemble those of NetrinA/B mutants. In embryos lacking NetrinA/B or frazzled, CNS axon commissures are partially missing or thinner, with the posterior commissure disrupted more severely than the anterior commissure. In addition, occasional breaks are observed in the longitudinal tracts. The Netrin double mutant and fra null mutants also show the same spectrum of motor axon projection abnormalities, particularly in the dorsal path of the ISN and in the SNb. Ectopic expression of the Drosophila Netrin genes, but not frazzled, leads to misrouting of CNS and motor axons that express Frazzled proteins, and the presence of Frazzled on motor axons ( $\mathrm{SNa}$ ) that normally do not innervate Netrin expressing targets can explain the responsiveness of these axons to ectopic Netrin expression.

From the binding of vertebrate netrin to DCC (KeinoMasu et al., 1996), the study of UNC-40 (Chan et al., 1996), and these data, we do not know whether DCClike proteins constitute a complete receptor-mediating netrin-dependent chemoattraction or a key ligand-binding component. Expression of Frazzled in axons that do not normally express it does not appear to cause guidance defects. Further analysis of frazzled and $\mathrm{Ne}-$ trins in Drosophila may help resolve this and other issues and identify additional components of this guidance system.

\section{Experimental Procedures}

\section{frazzled Cloning}

DNA flanking the 49B insertion was isolated by plasmid rescue (Pirotta, 1986) and used to isolate additional flanking DNA. Digoxygenin-labeled (Boehringer-Mannheim) DNA probes from these genomic clones were then used to identify transcripts in the region by in situ hybridization on embryos and to clone cDNAs from embryonic cDNA libraries. Multiple clones were isolated and sequenced on both strands to obtain the entire fra sequence. Corresponding genomic DNA fragments were sequenced to confirm the cDNA sequence and to identify intron/exon boundaries.

Isolation and Identification of fra Mutants

The P-element at 49B was mobilized by standard methods. $56 / 293$ excision lines were lethal; the lethal lines that failed to complement the 49B deficiency $D f(2 R) v g 135$ comprised a single complementation group: frazzled. Embryos 12-16 hr old from 20 frazzled mutant lines balanced over CyO, $P[w+, T 8-l a c Z]$ (Kolodziej et al., 1995) were screened by in situ hybridization with fra and lac $Z$ cDNAs. One line ( fra $^{1}$ ) lacking detectable fra transcripts in mutant embryos was identified, and subsequently shown by chromosome squashes to be a 2;3 translocation. EMS alleles were provided by Troy Zars and David Hyde or were identified in a screen for mutations affecting axon development in the CNS (Seeger et al., 1993). Mutations were analyzed by Southern blotting. DNA fragments of $\mathrm{fra}^{3}$ were sequenced after 24 rounds of PCR on single homozygous mutant embryos and the mutation was confirmed by sequencing three independent clones.

Production of Antisera, Histochemistry, and Microscopy To raise antisera against the C-terminal domain of Frazzled, PCRamplified DNA encoding the 278 amino acid Frazzled C-terminal domain (amino acid 1328 of the longer transcript to TAA) was cloned into ptrcC (Invitrogen) and $\sim 200 \mu \mathrm{g}$ of the (His) ${ }_{6}$ Frazzled fusion protein were isolated from $20 \mathrm{I}$ of culture after purification on polyhistidine affinity resin (Qiagen). Antibodies were raised against SDSPAGE purified fusion protein at Caltag Laboratories and further purified on protein-A agarose (Boehringer Mannheim). Histochemistry and microscopy have been previously described (Kolodziej et al., 1995).

\section{frazzled Rescue}

An EcoRI-Xbal partial digestion DNA fragment containing nucleotides 1-5038 from the shorter isoform of the frazzled cDNA was cloned into pGAL4-UAST (Brand and Perrimon, 1993). One homozygous insertion line (chromosome III) containing the resulting pUASfra was obtained. The GAL4 expressing panneural enhancer trap line 1407 was recombined with the $\mathrm{fra}^{3}$ mutation and balanced over the $\mathrm{CyO}^{276}$ chromosome. $\mathrm{CyO}^{276}$-containing embryos express lacZ in the embryonic salivary glands and hindgut. yw; $1407 \mathrm{fra}^{3} / \mathrm{CyO}^{276}$ females were then crossed to $y w ; B c$ Elp/CyO ${ }^{276} ; p U A S-f r a / T M 6 b$ $P[Y+]$ males, and males of genotype $1407 \mathrm{fra}^{3} / \mathrm{CyO}^{276}$; pUAS-fra/+ were crossed to $\mathrm{fra}^{4} / \mathrm{CyO}$-elav-lacZ females (or $\mathrm{fra}^{4} / \mathrm{CyO}^{276}$ females for the motor axon rescue experiment). CyO-elav-lacZ-containing embryos express $\beta$-galactosidase in all neurons. $1407 \mathrm{fra}^{3} / \mathrm{fra}^{4}$ adult flies containing the transgene were not recovered. Experiments using the GAL4 pan-muscle line $24 B$ were similarly designed. Dorsal muscle connectivity was scored under $250 \times$ or greater magnification.

\section{Acknowledgments}

The authors wish to thank Troy Zars and David Hyde for the fra ${ }^{2}$ and fra $^{3}$ mutant stocks, Hong Ao and Teresa Lavin for technical assistance, Barry Dickson for observing the translocation in $\mathrm{fra}^{1}, \mathrm{~K}$. Zinn and T. Kornberg for cDNA libraries, and J. Culotti and M. Tessier-Lavigne for communicating results prior to publication. K. J. M. received predoctoral support from the NSF, and $P$. A. K. received postdoctoral support from the NIMDH and the ACS, California Chapter. This work was supported by the Howard Hughes Medical Institute. C. S. G, L. Y. J., and Y. N. J. are Howard Hughes Investigators, and P. A. K. is a Howard Hughes Assistant Investigator.

Received July 19, 1996; revised September 9, 1996.

\section{References}

Altschul, S.F., Gish, W., Miller, W., Myers, E.W., and Lipman, D.J. (1990). Basic local alignment search tool. J. Mol. Biol. 215, 403-410. Bier, E., Vaessin, H., Shepherd, S., Lee, K., McCall, K., Barbel, S., Ackerman, L., Caretto, R., Uemura, T., Grell, E., Jan, L.Y., and Jan, Y.N. (1989). Searching for pattern and mutation in the Drosophila genome with a P-lacZ vector. Genes Dev. 3, 1273-1287.

Brand, A.H., and Perrimon, N. (1993). Targeted gene expression as a means of altering cell fates and generating dominant phenotypes. Development 118, 401-415.

Chan, S.S.-Y., Zheng, H., Su, M.W., Wilk, R., Killeen, M.T., 
Hedgecock, E.M., and Culotti, J.G. (1996). UNC-40, a C. elegans homolog of DCC (Deleted in Colorectal Cancer), is required in motile cells responding to UNC-6 netrin cues. Cell, this issue.

Chuong, C.M., Jiang, T.X., Yin, E., and Widelitz, R.B. (1994). cDCC (chicken homologue to a gene deleted in colorectal carcinoma) is an epithelial adhesion molecule expressed in the basal cells and involved in epithelial-mesenchymal interaction. Dev. Biol. 164, 383-397.

Colamarino, S.A., and Tessier-Lavigne, M. (1995). The axonal chemoattractant Netrin-1 is also a chemorepellent for trochlear motor axons. Cell 81, 621-629.

Fearon, E.R., Cho, K.R., Nigro, J.M., Kern, S.E., Simons, J.W., Ruppert, J.M., Hamilton, S.R., Preisinger, A.C., Thomas, G., Kinzler, K.W., and Vogelstein, B. (1990). Identification of a chromosome 18q gene that is altered in colorectal cancers. Science $247,49-56$.

Goodman, C.S. (1996). Mechanisms and molecules that control growth cone guidance. Annu. Rev. Neurosci. 19, 341-377.

Hamelin, M., Zhou, Y., Su, M.W., Scott, I.M., and Culotti, J.G. (1993). Expression of the UNC- 5 guidance receptor in the touch neurons of C. elegans steers their axons dorsally. Nature 364, 327-330.

Harris, R., Sabatelli, L.M., and Seeger, M.A. (1996). Guidance cues at the Drosophila CNS midline: identification and characterization of two Drosophila Netrin/UNC-6 homologs. Neuron 17, 217-218.

Hedgecock, E.M., Culotti, J.G., and Hall, D.H. (1990). The unc-5, unc- 6 , and unc- 40 genes guide circumferential migrations of pioneer axons and mesodermal cells on the epidermis in C. elegans. Neuron 4, 61-85.

Hedrick, L., Cho, K.R., Fearon, E.R., Wu, T.C., Kinzler, K.W., and Vogelstein, B. (1994). The DCC gene product in cellular differentiation and colorectal tumorigenesis. Genes Dev. 8, 1174-1183.

Higgins, D.G. (1994). CLUSTAL V: multiple alignment of DNA and protein sequences. Methods Mol. Biol. 25, 307-318.

Ishii, N., Wadsworth, W.G., Stern, B.D., Culotti, J.G., and Hedgecock, E.M. (1992). UNC-6, a laminin-related protein, guides cell and pioneer axon migrations in C. elegans. Neuron 9, 873-881.

Keino-Masu, K., Masu, M, Hinck, L., Leonardo, D.E., Chan, S., Culotti, J., and Tessier-Lavigne, M. (1996). Deleted in Colorectal Cancer $(D C C)$ encodes a netrin receptor. Cell, this issue.

Kennedy, T.E., Serafini, T., de la Torre, J.R., and Tessier-Lavigne, M. (1994). Netrins are diffusible chemotropic factors for commissural axons in the embryonic spinal cord. Cell 78, 425-435.

Kolodziej, P.A., Jan, L.Y., and Jan, Y.N. (1995). Mutations that affect the length, fasciculation, or ventral orientation of specific sensory axons in the Drosophila embryo. Neuron 15, 273-286.

Luo, L., Liao, Y.J., Jan, L.Y., and Jan, Y.N. (1994). Distinct morphogenetic functions of similar small GTPases: Drosophila Drac1 is involved in axonal outgrowth and myoblast fusion. Genes Dev. 8, 1787-1802.

Mitchell, K.J., Doyle, J.L., Serafini, T., Kennedy, T.E., TessierLavigne, M., Goodman, C.S., and Dickson, B.J. (1996). Genetic analysis of Netrin genes in Drosophila: netrins guide CNS commissural axons and peripheral motor axons. Neuron 17, 203-215.

Patthy, L. (1990). Homology of a domain of the growth hormone/ prolactin receptor family with type III modules of fibronectin [letter]. Cell 61, 13-14.

Pierceall, W.E., Cho, K.R., Getzenberg, R.H., Reale, M.A., Hedrick, L., Vogelstein, B., and Fearon, E.R. (1994). NIH3T3 cells expressing the Deleted in Colorectal Cancer (DCC) tumor suppressor gene product stimulate neurite outgrowth in rat PC12 pheochromocytoma cells. J. Cell. Biol. 124, 1017-1027.

Pirotta, V. (1986). Cloning Drosophila genes. In Drosophila: A Practical Approach, D.R. Roberts, ed. (Oxford: IRL Press), pp. 83-100.

Seeger, M., Tear, G., Ferres-Marco, D., and Goodman, C.S. (1993). Mutations affecting growth cone guidance in Drosophila: genes necessary for guidance toward or away from the midline. Neuron 10 , 409-426.

Serafini, T., Kennedy, T.E., Galko, M.J., Mirzayan, C., Jessell, T.M., and Tessier-Lavigne, M. (1994). The Netrins define a family of axon outgrowth-promoting proteins homologous to C. elegans UNC-6. Cell 78, 409-424.

Tessier-Lavigne, M. (1994). Axon guidance by diffusible repellants and attractants. Curr. Opin. Genet. Dev. 4, 596-601.

Van Vactor, D.V., Sink, H., Fambrough, D., Tsoo, R., and Goodman, C.S. (1993). Genes that control neuromuscular specificity in Drosophila. Cell 73, 1137-1153.

Vielmetter, J., Kayyem, J.F., Roman, J.M., and Dreyer, W.J. (1994). Neogenin, an avian cell surface protein expressed during terminal neuronal differentiation, is closely related to the human tumor suppressor molecule deleted in colorectal cancer. J. Cell Biol. 127, 2009-2020.

Wadsworth, W., Bhatt, H., and Hedgecock, E. (1996). Neuroglia and pioneer neurons express UNC-6 to provide global and local Netrin cues for guiding migrations in C. elegans. Neuron 16, 35-46.

Williams, A.F., and Barclay, A.N. (1988). The immunoglobulin superfamily-domains for cell surface recognition. Annu. Rev. Immunol. $6,381-405$.

\section{GenBank Accession Numbers}

The GenBank accession numbers for the sequences of the two frazzled isoforms are U71001 and U71002, respectively. 